\title{
Object-based attention: Sensory modulation or priority setting?
}

\author{
SARAH SHOMSTEIN and STEVEN YANTIS \\ Johns Hopkins University, Baltimore, Maryland
}

\begin{abstract}
The detection of an invalidly cued target is faster when it appears within a cued object than when it appears in an uncued object equally distant from the cued location; this is a manifestation of objectbased attention. Five experiments are reported in which it was investigated whether early sensory enhancement (in which attention "spreads" within an attended object but stops at its borders) or a later attentional prioritization mechanism best accounts for these effects. In Experiments 1-4, subjects identified a centrally located target with a buttonpress while attempting to ignore flanking distractors that were mapped to either a compatible or an incompatible response. The flankers appeared either within the object occupied by the target or in a different object but at the same distance from the target. The well-known effect of distance between the targetand the flankers on the magnitude of the compatibility effect was replicated. However, whether the target and the flankers were in the same or different objects had no effect on the magnitude of the compatibility effect. In Experiment 5, when attention could not be narrowly focused in advance, object-based modulation of the flanker effect was observed. These results suggest that object-based selection may reflect an object-specific attentional prioritization strategy, rather than object-based attentional modulation of an early sensory representation.
\end{abstract}

Until the early 1980 s, it was widely assumed that attention is typically directed to regions in space, in a manner that is analogous to a graded spotlight that illuminates a local convex region. One source of support for this view was the finding that the spatial separation between elements significantly modulates attentional effects (e.g., Downing \& Pinker, 1985; B. A. Eriksen \& C. W. Eriksen, 1974; C. W. Eriksen \& Hoffman, 1972; Hoffman \& Nelson, 1981; LaBerge, 1983; Posner, Snyder, \& Davidson, 1980; Yantis \& Johnston, 1990). B. A. Eriksen and C. W. Eriksen showed, for example, that the time required to identify a centrally located target letter depends on the identity of adjacent flanking letters: Flankers that are associated with the same response as the target (compatible flankers) speed response time (RT), relative to a neutral control condition, whereas flankers associated with a different response (incompatible flankers) slow RT, suggesting that subjects fail to completely ignore flankers. More important, this result provided evidence for a spatial account of attention, because it revealed that flanker interference is greatest when flankers are near to the target, as compared with when the flankers are farther away from the target.

Portions of this work were presented at the 1999 Object, Perception, and Memory meeting in Los Angeles. Support was provided by NIH Grant R01-MH43924 to S.Y. We thank Art Kramer, Bernhard Hommel, and an anonymous reviewer for helpful suggestions and Howard Egeth and Dominique Lamy for many fruitful discussions about this work. Correspondence concerning this article should be addressed to S. Shomstein, Department of Psychology, John Hopkins University, Baltimore, MD 21218 (e-mail: ssdw@jhu.edu).
Starting in the early 1980 s, evidence began to accumulate that some tasks engage a selective mechanism that operates on an object-based, rather than a location-based, representation (Kahneman \& Henik, 1981; Rock \& Guttman, 1981; for reviews, see Egeth \& Yantis, 1997, and Kanwisher \& Driver, 1992). For example, Duncan (1984) asked observers to view a briefly flashed display that contained a rectangle and a tilted line that were spatially superimposed. The rectangle was either tall or short, and it had a gap in its contour on either the right or the left side. The line was tilted either to the right or to the left, and it was either dashed or dotted. Observers were asked to report two dimensions on each trial (e.g., gap location and line tilt). Accuracy was greater when the subjects were required to report two attributes that belonged to the same object (e.g., the height and the gap location of the rectangle) than when they had to report one attribute from each object (e.g., the height of the rectangle and the tilt of the line). Because the two objects were superimposed and thus occupied overlapping spatial locations, Duncan argued that selection based only on spatial location could not account for the obtained results and that they therefore reflected an objectbased selection mechanism (for further discussion of Duncan, 1984, see Watt, 1984).

More recently, Egly, Driver, and Rafal (1994) introduced a new paradigm for studying both location-based and object-based selection. Two adjacent vertically or horizontally oriented rectangles appeared in the visual display. After several seconds, the contour at one end of one of the rectangles brightened briefly. This cued the observer to direct attention to that location while maintaining fixa- 
tion at the center of the display. The target event (a white square appearing at one end of one rectangle) was most likely to occur in the cued location (a validly cued target) but could also occur in the other end of the cued rectangle (an invalidly cued same-object target) or in the other rectangle, at the same distance from the cue (an invalidly cued differentobject target). Both location-based and object-based effects were observed. Detection was faster for validly cued targets than for invalidly cued ones, suggesting that the distance between the cued location and the target affects perceptual efficiency (a space-based effect). Furthermore, when the cue was invalid, same-object targets were detected more rapidly than different-object targets (an object-based effect). The latter result suggests that when part of an object is attended, the rest of the object benefits perceptually. This paradigm has been extended in several subsequent studies (e.g., Behrmann, Zemel, \& Mozer, 1998; Moore, Yantis, \& Vaughan, 1998; Watson \& Kramer, 1999).

At least two possible mechanisms for attentional modulation of perceptual efficiency can be invoked to account for these results. The first, which we term sensory enhancement, is that the deployment of attention improves the quality of an early sensory representation. Such an improvement can be viewed as a result of biased competition among neural representations (Desimone \& Duncan, 1995). According to this view, early sensory representations of multiple objects are mutually inhibitory, so that the strength of a representation is weaker when an object is presented in a multielement scene than when it is presented alone (Kastner, De-Weered, Desimone, \& Ungerleider, 1998; Reynolds, Chelazzi, \& Desimone, 1999). A top-down biasing signal that is evoked by a spatial cue or by task instructions can strengthen one of these representations, which will cause it to more effectively compete with neighboring representations. When an object "wins" the competition, it may be said to have been selected. To the extent that the top-down biasing signal is spatially imprecise (owing, e.g., to the limited spatial resolution of receptive fields at various levels of the visual pathways; see Reynolds et al., 1999, for a discussion), top-down facilitation might spread within a local region, falling off with distance from the center of the attended region. This could account for distance effects as measured by both speed and accuracy in cued-attention paradigms.

Object-based attentional modulation of early sensory representations could be viewed as a spatial spread of topdown facilitation that respects object boundaries, so that representations within an attended object are stronger than representations outside the attended object. It should be noted that although there is a straightforward explanation for distance effects in this account (it is a result of the retinotopic organization of early vision and of the increasing size of receptive fields in successively later stages of the visual pathway), there is no correspondingly obvious explanation of how scene segmentation could affect the top-down biasing signal and thereby influence the spread of attention in an object-based fashion. Presumably, some form of feedback from higher object representations to lower feature representations would be required.

A second possible mechanism for improved efficiency in visual selection paradigms, which we term attentionalprioritization, is a later process that affects the order in which different regions of the scene are visually investigated when multiple attentional "glimpses" are required. ${ }^{1}$ According to this idea, there is a predisposition to assign higher priority to locations within an already attended object than to locations in other objects.

Many of the studies in which object-based attentional modulation has been observed have employed paradigms that require attention to be deployed to multiple parts of the scene over time (see the General Discussion section for exceptions to this generalization). For example, in cuing paradigms (e.g., Egly et al., 1994; Moore et al., 1998), subjects first examine the cued location, and if the target is not found, they may be strategically inclined to assign greater priority to other locations within the cued object, so that the items within the cued object are examined before items in the uncued object. Avrahami (1999) showed that there is a tendency for observers to direct attention from a cued location along contours to uncued locations when there is no incentive to do so, even when the contours do not bound closed objects. ${ }^{2}$ Such strategic scanning would yield the sort of object-specific benefits that were reported by Egly et al. (1994) and others. In studies that employed matching or counting tasks (Behrmann et al., 1998; Watson \& Kramer, 1999), the nature of the task is such that subjects must seek pairs of task-relevant features for comparison, and as in the studies described above, they may adopt an (explicit or implicit) strategy to investigate features that are in or part of one object before moving on to those within other objects. It is useful to view this account in the context of Wolfe's (1994) Guided Search model: The assignment of attentional priorities that govern the order of search may well be affected not only by local feature contrast and by similarity to a target template (Cave \& Wolfe, 1990; Wolfe, 1994), but also by object structure.

Object-based attentional prioritization affects only the order of search, and not the quality of the perceptual representation at different locations in the scene. It is thus quite different from early sensory enhancement that respects object boundaries. This latter view, however, in which attention "spreads" within an object but stops at its boundaries, is implicit in many current object-based accounts.

Moore, Yantis, Vaughan, and Handwerker (2001) recently reported evidence that object-based attentional modulation does not result from early sensory enhancement that respects object boundaries. They employed a cued discrimination task like the one used by Egly et al. (1994) and by Moore et al. (1998). Two versions of the task were carried out. The first was an RT version that matched the no-occluder condition of Moore et al. (1998). The second was an accuracy version in which the stimuli were presented briefly and then masked. The critical stimulus-mask onset asyn- 
chrony required for $85 \%$ accuracy was estimated with a staircase tracking procedure to provide a measure of the quality of the sensory representation separately for the cued, uncued same-object, and uncued different-object targets. The results of the RT version of the experiment revealed the standard object-specific advantage. However, in the accuracy version of the task, no object-specific advantage was observed. Moore et al. (2001) argued that directing attention to the cued location in the accuracy version of the task produced sensory facilitation that fell off with the distance from the cued location-in all directions, regardless of object boundaries. This deployment of attention did not selectively augment the quality of the perceptual representation at locations within the cued object relative to equally distant locations in other objects. They attributed the object-specific advantage observed in the RT version of the task to an object-based search strategy in which locations within an attended object are investigated before locations in other objects-a strategy that is available only when the stimuli are visible for an extended period.

In the present paper, we further investigate the extent to which object-based attentional modulation is a manifestation of early sensory enhancement that respects object boundaries or of an attentional prioritization strategy that investigates locations within an already attended object before other locations. We employed a modified flanker compatibility paradigm (after B. A. Eriksen \& C. W. Eriksen, 1974). In Experiments 1-4, subjects were instructed to attend to the center of a scene containing three rectangles (see Figure 1). Three letters then appeared: one in the central attended location and two flanking that location (to the left and right or above and below). The flankers appeared either within the same rectangle as that occupied by the target or in different rectangles. The subjects identified the centrally located target item with a buttonpress while attempting to ignore the flanking distractors.

Because the target was presented only in the center, optimal performance required all noncentral locations to be ignored. If attention "spreads" within attended objects and stops at their borders, thereby selectively strengthening early sensory representations within the attended object (as compared with representations of elements within unattended objects), flankers within the attended object should have a greater influence on performance (thus yielding larger flanker compatibility effects) than do flankers in unattended objects. In contrast, if the spread of attention does not respect object boundaries and object-based attentional modulation reflects the order in which locations in the scene are investigated, there should be little if any effect of whether the flankers are in the attended or the unattended rectangles (because the target occupies a known
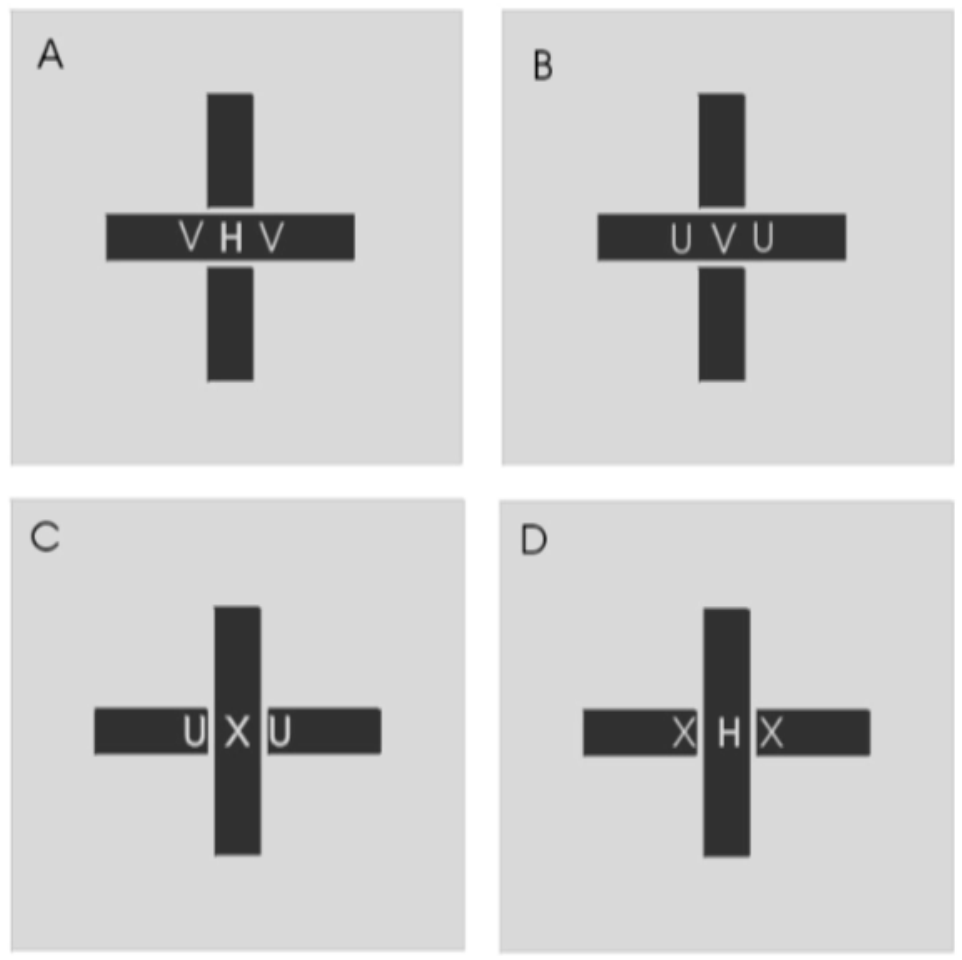

Figure 1. Example display for each of the four conditions. $H$ or $V$ required a left response; $\mathrm{V}$ or $\mathrm{X}$ required a right response. (A) Same-object compatible response; (B) same-object incompatible response; (C) different-object compatible response; (D) different-object incompatible response. Flanker distance remained constant among conditions. 
location and no search of the display is required). To anticipate, no object-specific modulation of the flanker compatibility effect was observed in Experiments 1-4, thus supporting an attentional prioritization account.

In Experiment 5, a modification of the paradigm used in Experiments 1-4 was used in which target location uncertainty was introduced and observers were required to direct attention to multiple locations within the scene. Under these circumstances, in which attentional prioritization could operate, object-specific modulation of the flanker effect was observed, confirming that the paradigm is sufficiently sensitive to reveal such modulation when it is present.

\section{EXPERIMENT 1}

\section{Method}

Subjects. Sixteen subjects were recruited from the Johns Hopkins University community. All the subjects reported normal or corrected-to-normal visual acuity and were naive as to the purpose of the experiment. The subjects were paid $\$ 7$ for their participation in a single 50-min session.

Apparatus and Stimuli. Stimuli were displayed on a 19-in. Taxan color monitor driven by an Artist Graphics XJS-1280 graphics accelerator board. A chinrest was used to ensure that the observer's eyes were positioned $62 \mathrm{~cm}$ from the screen. A custom response box was used to collect buttonpress responses. Figure 1 shows the layout of some typical displays for Experiment 1 . The display consisted of three solid red rectangles, with a luminance of $3.5 \mathrm{~cd} / \mathrm{m}^{2}$, presented on a square gray background region with a luminance of $7.0 \mathrm{~cd} / \mathrm{m}^{2}$. The gray background square subtended $8^{\circ} \times$ $8^{\circ}$ of visual angle. The large central rectangle subtended visual angles of $2.8^{\circ} \times 0.5^{\circ}$, and the smaller flanking rectangles each subtended visual angles of $1^{\circ} \times 0.5^{\circ}$. The two small rectangles were separated from the long rectangle by a gap subtending a visual angle of $0.1^{\circ}$. The long rectangle was oriented horizontally on a randomly selected half of the trials and vertically on the remaining half.

A target letter always appeared at the center of the display, whereas flanking letters appeared adjacent to the target. Each letter subtended $0.37^{\circ} \times 0.37^{\circ}$ of visual angle. The flankers were positioned $0.18^{\circ}$ from the target, edge to edge, a distance that has been shown to yield robust flanker compatibility effects (e.g., B. A. Eriksen \& C. W. Eriksen, 1974). The letters were the color of the gray background square.

Design and Procedure. A $2 \times 2$ within-subjects factorial design was used, with target/flanker compatibility (compatible or incompatible) and target-flanker relation (same or different objects) as factors. The target and flankers were considered compatible if they all mapped onto the same response key and incompatible if they mapped onto different response keys (in the compatible condition, the target and flankers were always different letters). Each of the four combinations of conditions occurred equally often. Rectangle orientation and target and distractor identity were selected randomly and equally often within blocks, within the constraints of the experimental design. Targets $\mathrm{H}$ and $\mathrm{V}$ mapped onto the left response key, and $\mathrm{U}$ and $\mathrm{X}$ mapped onto the right response key.

Each trial began with the gray background presented for a 2,000msec intertrial interval (ITI). The three rectangles were then presented on the screen for $1,000 \mathrm{msec}$, and then the target and the flankers appeared. The stimuli remained on the screen until the subject responded. The subjects were instructed to respond as quickly as possible while making no more than about $5 \%$ errors. Error feedback consisted of a beep and an increase in the ITI of 2,000 msec following that trial. Each subject completed one 32-trial practice block and
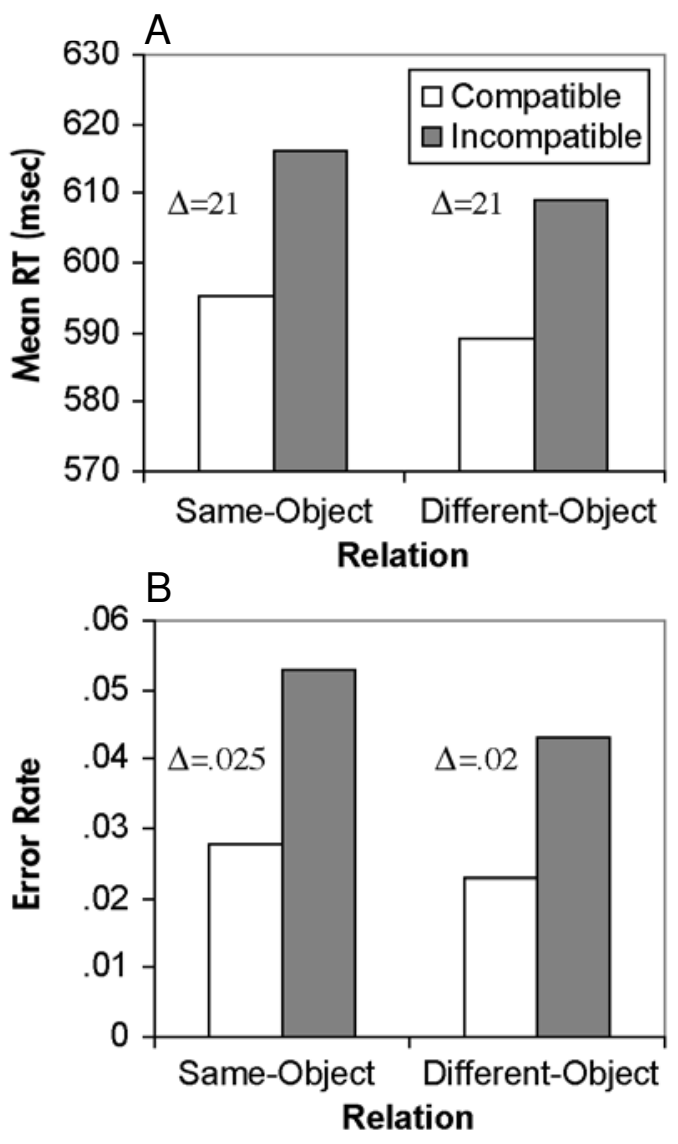

Figure 2. Mean correct response times (A) and error rates (B) in Experiment 1. The data are collapsed across the vertical and horizontal orientations of the display.

15 blocks of 32 experimental trials. The subjects received feedback after each block.

\section{Results}

Only the RTs for correct responses were analyzed. Figure 2 shows the mean correct RT and the mean error rate for each condition of Experiment 1. Preliminary analysis indicated that there was no main effect of the orientation of the rectangles and no interaction of orientation with any of the other factors (all $F_{\mathrm{s}}<1$ ), so the data were collapsed across the vertical and horizontal orientations.

A repeated measures analysis of variance (ANOVA) was conducted, with compatibility and relation as withinsubjects factors and RT as the dependent measure. The ANOVA revealed a significant main effect of compatibility $[F(1,15)=29.39, p<.001]$ : RTs for compatible flankers $(M=592 \mathrm{msec})$ were faster than those for incompatible ones $(M=612 \mathrm{msec})$. In addition, there was a significant main effect of relation $[F(1,15)=5.68, p=.05]$, so that RTs were slightly slower when the target and flankers appeared in the same object $(M=605 \mathrm{msec})$ than when they appeared in different objects $(M=599 \mathrm{msec})$. However, the interaction between compatibility and target-flanker relation was not significant $[F(1,15)<1]$. The flanker in- 


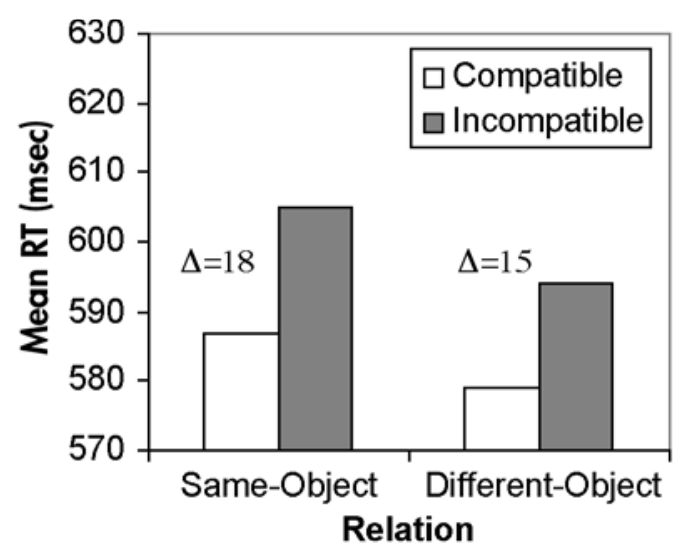

Figure 3. Mean correct response time (RT) results in Experiment 2. Flankers in this experiment were positioned an intermediate distance away from the target.

terference effect of $21 \mathrm{msec}$ did not depend on whether the target and flankers were within the same object or not.

An analysis of the error rates revealed a similar pattern. The main effect of compatibility was significant $[F(1,15)=$ $23.7, p<.001]$, and the main effect of relation was not significant $[F(1,15)=3.34, p<1]$. In addition, there was no significant interaction between the factors. All of the subjects performed with at least $90 \%$ accuracy in each condition in all of the experiments.

\section{Discussion}

The results of Experiment 1 replicate earlier studies showing that subjects respond faster when targets and flankers are compatible than when they are incompatible. However, they fail to reveal an object-based modulation of the flanker effect.

Responses were slightly slower when all the letters appeared within the same object than when they appeared in different objects. We speculate that this result may reflect a segmentation process that is required to separate irrelevant information (i.e., flankers) from the relevant information (i.e., the target) when the items are within the same object; this segmentation stage may not be required when the flankers appear in different objects, because they are already perceptually segmented from the target. This was a very small effect and so must be interpreted with caution. The primary result of Experiment 1, however, was that the flanker compatibility effect was not affected by whether the items appeared within the same object or not.

\section{EXPERIMENTS 2 AND 3}

It might be argued that the absence of an object-based attentional modulation effect in Experiment 1 can be attributed to a lack of statistical power (although the effect size was, in fact, zero). To rule out this possibility, we introduced a distance manipulation to exploit the fact that the farther away from the target the flankers are, the smaller the interference they produce, at least within a cer- tain range of distances (e.g., B. A. Eriksen \& C. W. Eriksen, 1974; Hoffman \& Nelson, 1981; Posner, 1980). This allowed us to determine whether our paradigm was powerful enough to reveal any modulation of the flanker compatibility effect.

It is also possible that object-based attentional modulation is overridden by space-based modulation when flankers are very close to the target. In Experiment 1, the flankers were only $0.18^{\circ}$ from the target. Therefore, in Experiment 2 we tested for object-based attentional modulation with the flankers positioned farther away from the target, and in Experiment 3 we manipulated target-flanker distance within subjects.

\section{Method}

Subjects. Sixteen new subjects from the Johns Hopkins University community participated in Experiment 2, and 19 new subjects participated in Experiment 3. All the subjects reported normal or corrected-to-normal visual acuity and were naive as to the purpose of the experiment. The subjects were paid $\$ 7$ for their participation.

Apparatus and Stimuli. The stimuli were the same as those in Experiment 1, with one difference. Flankers in Experiment 2 appeared at an intermediate distance from the target $\left(0.37^{\circ}\right.$ of visual angle). In Experiment 3, flankers appeared at two distances from the target. The near distance was $0.18^{\circ}$ (as in Experiment 1), and the far distance was $0.69^{\circ}$.

Design and Procedure. The design of Experiment 2 was the same as that in Experiment 1. For Experiment 3, a $2 \times 2 \times 2$ withinsubjects design was used. The factors were target/distractor compatibility (compatible, incompatible), target-distractor relation (same-object, different-object), and distance (near, far). Rectangle orientation and target and distractor identity were selected randomly and equally often within blocks. The trial sequence was identical to that in Experiment 1. Each subject completed 32 practice trials and eight blocks of 64 experimental trials.

\section{Results}

Figures 3 and 4 show the mean correct RTs for Experiments 2 and 3, respectively. These data were collapsed across the two rectangle orientations (vertical and horizontal), because none of the main effects or interactions involving that factor was significant (all $\left.F_{\mathrm{S}}<1\right)$.

Experiment 2. The data were subjected to a two-way ANOVA, with relation and compatibility as within-subjects factors and RT and error rate as dependent measures. The ANOVA revealed a significant main effect of compatibility $[F(1,15)=15.24, p<.001]$ : RTs were faster for compatible than for incompatible flankers. However, the interaction between compatibility and relation was not significant $[F(1,15)<1]$. The analysis of the error rates revealed the same pattern.

Experiment 3. An ANOVA was conducted, with relation, compatibility, and distance as within-subjects factors and RT and error rate as dependent measures. The ANOVA revealed a significant main effect of compatibility $[F(1,18)=$ $3.6, p<.001]$ and of distance $[F(1,18)=22.0, p<.001]$. RTs were faster when flankers appeared further away from the target $(M=594 \mathrm{msec})$ than when they appeared closer $(M=608 \mathrm{msec})$.

In addition, the ANOVA revealed two significant interactions: relation with distance, $[F(1,18)=6.8, p=.018]$ 
Table 1

Experiment 3: Mean Error Rates

\begin{tabular}{lccccc}
\hline & \multicolumn{2}{c}{ Same Object } & & \multicolumn{2}{c}{ Different Object } \\
\cline { 2 - 3 } \cline { 5 - 6 } Condition & Near & Far & & Near & Far \\
\hline Compatible & .01 & .02 & & .02 & .01 \\
Incompatible & .04 & .03 & & .03 & .02 \\
\hline
\end{tabular}

and compatibility with distance $[F(1,18)=4.5, p=.05]$. However, there was no significant three-way interaction observed $[F(1,18)=0.02$, n.s.]. Analysis of error rates (see Table 1) revealed a significant main effect of compatibility $[F(1,18)=17.4, p=.001]$ and a marginally significant main effect of relation $[F(1,18)=3.8, p=.07]$ and distance $[F(1,18)=3.6, p=.07]$. However, there were no significant interactions.

\section{Discussion}

The results obtained in Experiments 2 and 3 reveal space-based, but not object-based, modulation of the flanker compatibility effect. The compatibility effect was greater when the flankers were closer to the target than when they were farther away. This confirms that our paradigm is powerful enough to reveal modulations of the flanker compatibility effect when they are present. However, no objectspecific modulation of the compatibilityeffect was observed at any distance, as was evidenced by the absence of significant interactions involving compatibility and relation.

\section{EXPERIMENT 4}

It is important, of course, that any test of object-based modulations of the flanker effect employ perceptually distinct objects. One could argue that the objects used in Experiments 1-3 were too similar in appearance (they were the same color and texture) and too close together (the gap between the rectangles was only $0.1^{\circ}$, to permit us to use the very close distances initially used by B. A. Eriksen \& C. W. Eriksen, 1974). It might have been the case that the subjects perceived the three rectangles as one red cross. ${ }^{3}$ If so, it would not be surprising that no object-based attentional modulations were observed in Experiments 1-3.

In Experiment 4, we used a red central rectangle and black flanking rectangles to increase their distinctiveness. In addition, we manipulated the distance between the rectangles while maintaining a constant Euclidean distance between the target and the flankers.

\section{Method}

Subjects. Eleven new subjects from Johns Hopkins University participated in this experiment. All the subjects reported normal or corrected-to-normal visual acuity and were naive as to the purpose of the experiment. The subjects were paid $\$ 7$ for their participation.

Apparatus and Stimuli. The stimuli were the same as those in Experiment 2, with two differences. First, the color of the smaller rectangles was changed from red to black. Second, the gap between the central and the flanking rectangles was either small $\left(0.1^{\circ}\right.$, as in Experiments $1-3)$ or large $\left(0.25^{\circ}\right.$, or half the width of the rectangles). The flankers were positioned $0.37^{\circ}$ from the target throughout the experiment. Larger gaps were not used because the magnitude of the flanker effect declines dramatically with distance (e.g., the mean effect in Experiment 3 with a distance of $0.69^{\circ}$, although significant, was only $12 \mathrm{msec}$, an effect size that is similar to that reported by B. A. Eriksen \& C. W. Eriksen, 1974, for a corresponding distance), and this flanker distance would have compromised the power of the experiment. The fact that the large red and small black rectangles were different objects, particularly in the large-gap condition, was clear and unambiguous.

Design and Procedure. A $2 \times 2 \times 2$ within-subjects design was used, with compatibility (compatible, incompatible), relation (same object, different object), and rectangle gap (small, large) as factors. The procedure was identical to that in the previous experiments. Each subject completed 32 practice trials and five blocks of 128 experimental trials.

\section{Results and Discussion}

Figure 5 shows the mean correct RTs for Experiment 4. These data were collapsed across the two display orientations (vertical and horizontal), because none of the main effects or interactions involving that factor was significant (all $F_{\mathrm{s}}<1$ ).

An ANOVA was conducted, with relation, compatibility, and gap distance as within-subjects factors and RT and error rate as dependent measures. The ANOVA revealed a significant main effect of compatibility $[F(1,10)=29.0$, $p<.001]$. However, none of the other main effects and none of the two-way or three-way interactions was significant (all $F_{\mathrm{s}}<1$, n.s.). Analysis of error rates revealed only a significant main effect of compatibility; there were no significant interactions.
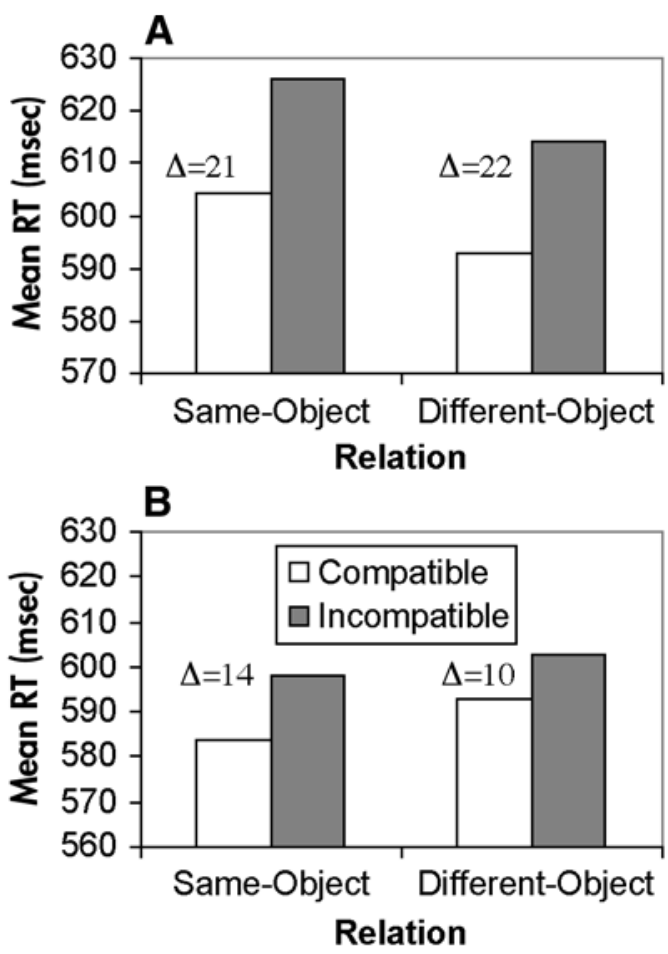

Figure 4. Mean correct response times (RTs) in Experiment 3. (A) Flankers presented at a near $\left(0.18^{\circ}\right)$ distance away from the target; (B) Flankers presented at a far $\left(0.69^{\circ}\right)$ distance away from the target. 

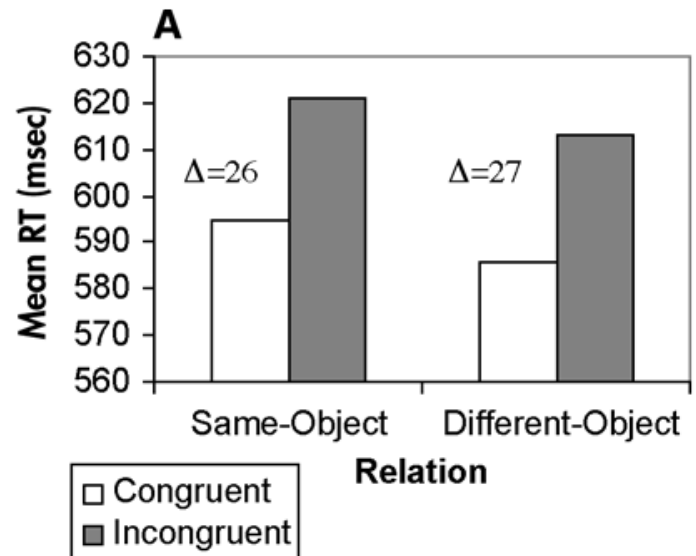

B

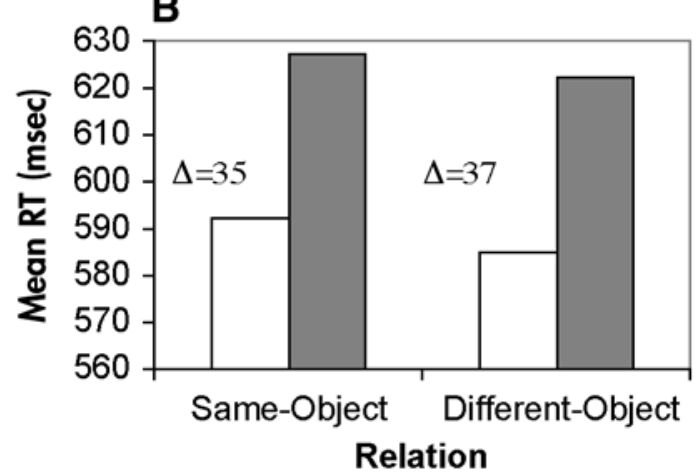

Figure 5. Mean correct response times (RTs) in Experiment 4. (A) Rectangle gap presented at the distance of $0.1^{\circ}$, as in Experiments 1, 2, and 3; (B) Rectangle gap increased to $0.25^{\circ}$ of visual angle away from the central rectangle.

The results of Experiment 4 show that increasing the distinctiveness of the rectangles by varying their color and their separation did not change the main finding in Experiments 1-3. Flanker interference is not affected by whether the target and the flankers are in the same or different objects, even when the objects in question are quite distinct.

\section{EXPERIMENT 5}

So far, we have demonstrated that under conditions of narrowly focused attention, the flanker compatibility effect is not influenced differentially by flankers appearing on the same or different objects (this finding has been corroborated by Lamy \& Egeth, in press, Experiment 4). Instead, early sensory enhancement of attended and nearby location does not respect object boundaries. According to the attentional prioritization account, when attention cannot be narrowly focused because multiple locations in the scene are task relevant (here, the target could appear in one of four locations), object-based effects should emerge. Such an outcome would show that it was the attentive state of the observer, and not some specific details of our paradigm, that determines whether object-based effects are observed.
In Experiment 5, a small bright square was flashed briefly in the center of the central rectangle (see Figure 6). The square was uninformative about the location of the upcoming target but was presumed to summon attention to the central rectangle. After a $100-m s e c$ interstimulus interval (ISI), the target and three nontargets appeared. The target appeared either in the cued rectangle or in one of the uncued rectangles. The two flankers never appeared in the same rectangle as the target (a third, nonsense symbol was presented to balance the display and prevent apparent motion effects).

The crucial factor here is whether the target appears in the cued object or in one of the uncued objects. The flanker compatibility effect should be small when the target appears in the cued object and the flankers are in the uncued object, because (according to the attentional prioritization account), when attention cannot be narrowly focused, locations within the cued object should have higher priority than locations in other objects. Conversely, the compatibility effect should be large when the target is in the uncued object and the flankers are in the cued object, because (according to this account) one or both of the flankers should be queried before the target (see note 1).

\section{Method}

Subjects. Twenty new subjects from Johns Hopkins University participated in this experiment. As in the previous experiments, the subjects reported normal or corrected-to-normal visual acuity and were naive as to the purpose of the experiment. The subjects participated in return for extra credit applied to a psychology course.

Apparatus and Stimuli. The stimuli were the same as those in Experiment 2, with two differences. First, a small bright square subtending a visual angle of $0.3^{\circ} \times 0.3^{\circ}$ was presented in the center of the display. In addition to two flankers, another character (e.g., a star) was presented in order to balance the display and prevent apparent motion. The star was easily discriminable from the letters and subtended $0.37^{\circ} \times 0.37^{\circ}$ of visual angle.

Although the precise location of the target was not known to the subject in advance of each trial, the target was known to appear in one of only four possible target locations, at an intermediate distance from the central cued location. On any given trial, the flankers and the nonletter character all appeared either near $\left(0.18^{\circ}\right)$ the cued location or far $\left(0.69^{\circ}\right)$ from the cued location.

Design and Procedure. A $2 \times 2 \times 2$ within-subjects design was used, with compatibility (compatible, incompatible), relation between the cued rectangle and the target rectangle (same object, different object), and flanker distance (near, far) as factors. Note that in this experiment, the targets and the flankers were never in the same object. The crucial issue was whether the target was in the cued object or not; in the latter case, the flankers necessarily appeared in the cued object.

The procedure was identical to that in the previous experiments, with several exceptions. First, after the initial configuration was presented on the screen for $1,000 \mathrm{msec}$, the spatially uninformative cue was flashed for $100 \mathrm{msec}$. After an ISI of $100 \mathrm{msec}$, the target, along with two flankers and a star, was presented. The target appeared in one of four locations that were located an intermediate distance away from the center of the display, with a nonsense character presented across from it along with two distractors (i.e., flankers) presented on the sides (see Figure 6). Distractors on any given trial appeared the same distance away from the center. The items remained on the screen until the response. Each subject completed one 32-trial practice block and eight blocks of 64 experimental trials. 

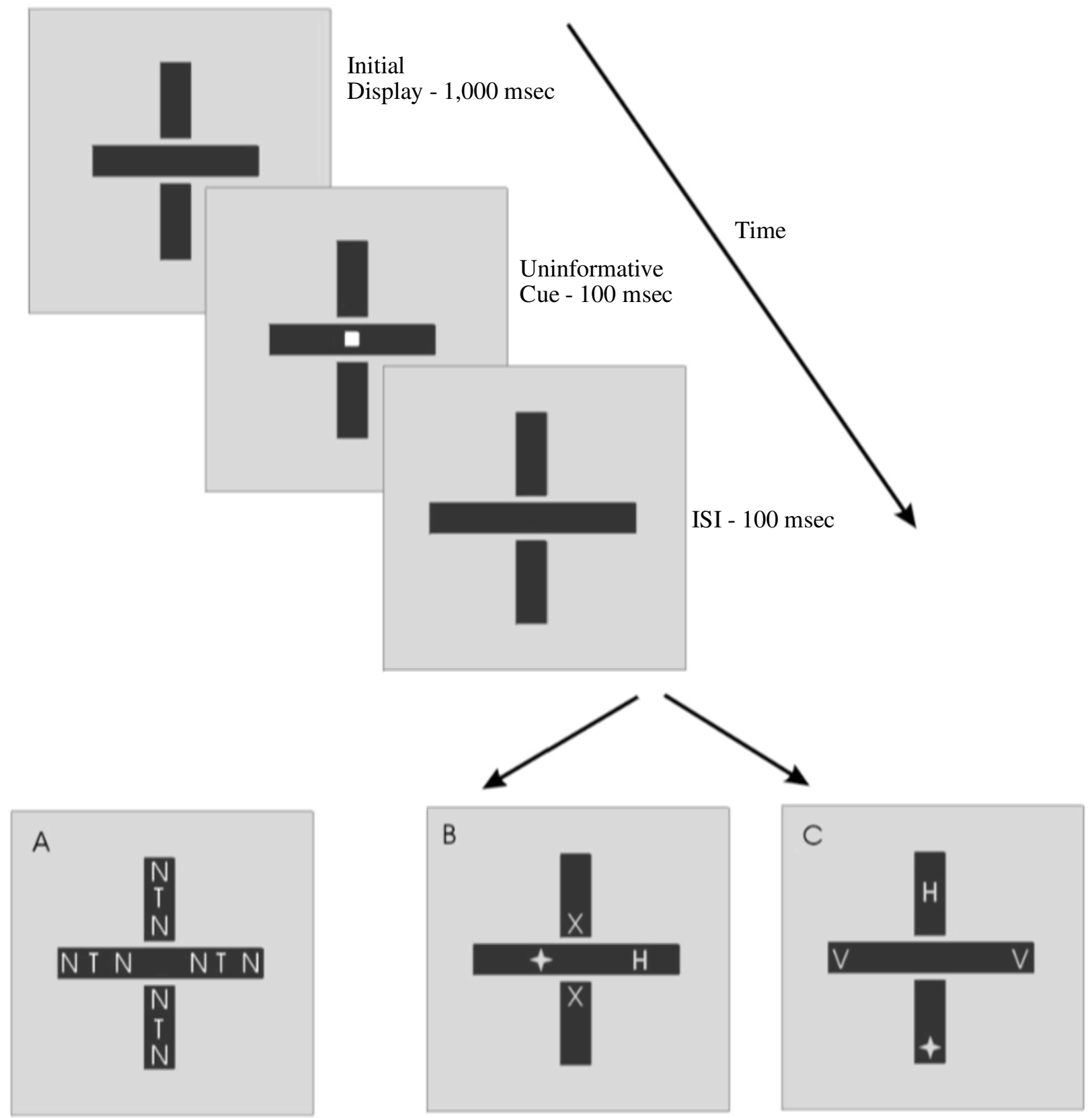

Figure 6. Sample trial from Experiment 5. As in prior experiments, $H$ or $V$ required a left response, whereas $U$ or $X$ required a right buttonpress. (A) A target-nontarget location map. The target could appear in one of the locations that are marked with a letter T. Nontargets could appear in the locations marked with a letter $N$, with the constraints that within a trial, (1) all nontargets were equidistant from the center of the display and (2) nontargets never appeared in the same object as the target (see the Method section for details). This map was not visible to the subjects. (B) A sample display from the cued object, incompatible response, near-distance condition. (C) A sample display from the uncued object, compatible response, far-distance condition.

\section{Results and Discussion}

Only RTs for correct responses that were less than $1,500 \mathrm{msec}$ were analyzed. Figure 7 shows the mean correct RTs for the near and far conditions in Experiment 5. The ANOVA was conducted, with compatibility, relation, and distance as within-subjects factors and RT as a de- pendent measure. The ANOVA revealed a significant main effect of compatibility $[F(1,19)=6.76, p<.02]$ : RTs for compatible flankers were faster $(M=836 \mathrm{msec})$ than those for incompatibleflankers $(M=849 \mathrm{msec})$. The main effect for distance was not significant $[F(1,19)=3.02, p=.09]$ : RTs for near flankers were slightly, but not significantly, 

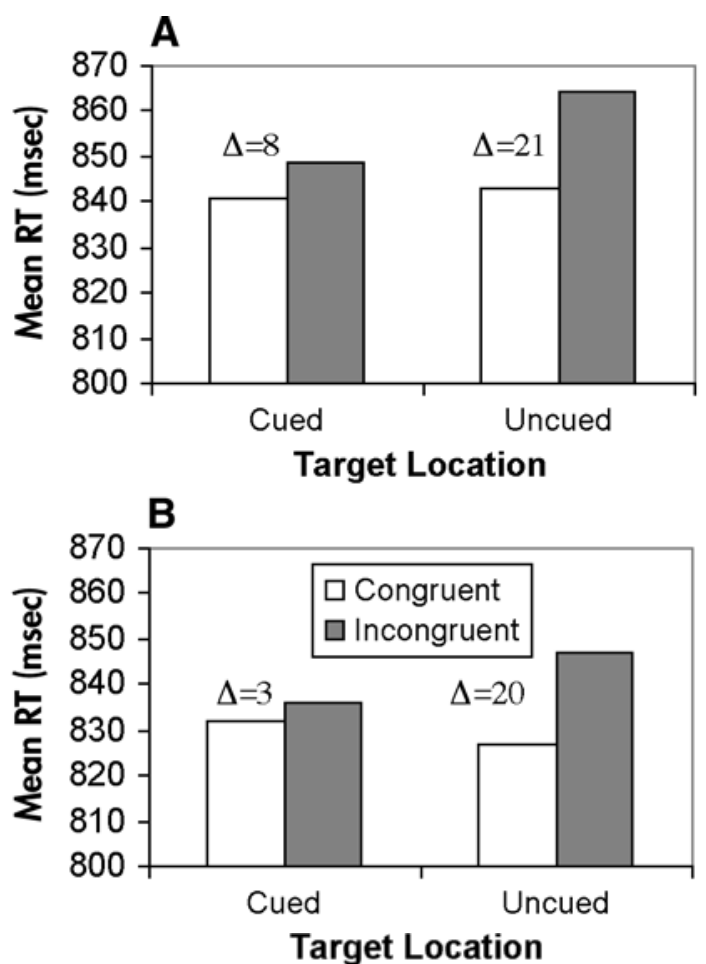

Figure 7. Mean correct response times (RTs) in Experiment 5. (A) Flankers presented at a near $\left(0.18^{\circ}\right)$ distance away from the center of the display. (B) Flankers presented at a far $\left(0.69^{\circ}\right)$ distance away from the center of the display. Note that when the target is in the cued object, the flankers are in the uncued objects, and vice versa.

slower than those for far flankers (this effect was significant in the error rate analysis; see below). In addition, there was a significant interaction between relation and compatibility $[F(1,19)=7.2, p<.02]$ : The compatibility effect when the target was in the cued object $(6 \mathrm{msec})$ was significantly smaller than when the target was in the uncued object $(21 \mathrm{msec})$. No other interaction was significant.

Note that RTs to targets in the compatible condition are no slower when the target is on the uncued object than when it is on the cued object (see Figure 5). One might have expected to find a reorientation cost when attention had to be redirected from the cued to the uncued rectangle (as observed, e.g., by Egly et al., 1994). One contravening factor here is that when attention must be redirected to the uncued rectangle, a compatible flanker is first encountered. A compatible flanker will contribute to preparation of the appropriate response, producing a "head start" in processing the eventual target. This may well have contributed to the absence of a reorientation cost.

An analysis of the error rates revealed a significant main effect of relation $[F(1,19)=5.46, p<.04]$, so that more errors were made in the different-object condition, as compared with the same-object condition. In addition, there was a significant main effect of distance $[F(1,19)=4.13$, $p=.05]$, so that error rates for the near distance were slightly greater than those for the far distance. There were no significant interactions among the factors.

These results are important to our argument for two reasons. First, they demonstrate that our paradigm is sensitive enough to detect object-based modulation of the flanker compatibility effect when it is present. Second, they show that the critical factor that determines whether object-based modulation will occur is whether attention must be directed to multiple locations in the scene, in which case an object-based attentional prioritization strategy causes attention to visit locations within the attended object before locations in other objects.

\section{GENERAL DISCUSSION}

The results from Experiments 1-4 revealed a complete absence of object-specific modulation of the flanker compatibility effect. Furthermore, consistent effects of targetflanker distance were observed (i.e., the effect was smaller when distractors were farther away from the target), showing that our paradigm was powerful enough to reflect spatial modulations of the flanker compatibility effect. Experiment 5 demonstrated that when attention had to be deployed to multiple locations in the scene, owing to spatial uncertainty, object-based attentional prioritization could operate to produce object-based modulation of the flanker effect. We conclude that the object-based effects observed in many selective attention paradigms may be attributable to an object-specific prioritization process and are not due to a "spread" of attention that respects object boundaries.

This conclusion can readily account for object-based effects in a variety of paradigms, such as those used by Behrmann et al. (1998), Egly et al. (1994), Moore et al. (1998), Moore et al. (2001), and Watson and Kramer (1999). How well does this view account for other sources of evidence for object-based attention? One of these is Duncan's (1984) study, in which he used a paradigm that resembles the accuracy version of Moore et al. (2001) in that it involved brief, masked displays and accuracy measures. According to the sensory enhancement mechanism, such displays should not provide the opportunity for the deployment of attention to multiple locations, which, according to the present account, is how object-based effects arise. At first glance, then, this experiment appears to present problems for the attentional prioritization account.

In our view, however, the object-based effects observed by Duncan (1984) are of a sort that is conceptually distinct from those revealed by paradigms like that of Egly et al. (1994). Indeed, Duncan interpreted his results as reflecting just the sort of attentional prioritization process outlined by Moore et al. (2001). According to Duncan, observers select one of the objects (including all of its relevant attributes) in a single selective episode. If the task requires judgments about two attributes of a single object, both of them would be available for report. However, if the task re- 
quires report of one attribute from each object, the second object has to be selected, and because of the rapidly following mask, this was frequently interrupted, thereby reducing accuracy. Thus, Duncan's explanation is perfectly consistent with a prioritization account of object-based effects.

This interpretation highlights an important property of experiments like that of Egly et al. (1994). When subjects are cued to attend to one end of one of the rectangles in preparation for an upcoming target, they do not select the rectangle as a whole. The judgmentrequired by the task did not concern the rectangle; it concerned a localized event in a location that happened to be defined relative to the rectangle. Egly et al. interpreted the validity effects in terms of the extra time required to shift attention from the cued location to another location, either within that rectangle (which could be done efficiently) or in another object (which incurred an additional between-objects cost). Similarly, in the experiment of Moore et al. (1998), the task required making a letter discrimination (rotated T vs. rotated L), not a decision about the rectangle as a whole. The fact that invalid same-object RTs were slower than valid RTs suggests that it was not the case that all locations in the rectangle were selected at once.

Another group of studies that has provided evidence for object-based selection but that does not appear to be consistent with our notion of attentional prioritization are those that have examined the influence of Gestalt grouping principles on the magnitude of the B. A. Eriksen and C. W. Eriksen (1974) flanker compatibility effect (Baylis \& Driver, 1992; Driver \& Baylis, 1989; Harms \& Bundesen, 1983; Kramer \& Jacobson, 1991). The rationale for these studies was that any modulation of the flanker effect by factors that play a role in perceptual grouping (e.g., similarity, common fate, uniform connectedness) would constitute evidence for object-based selection. ${ }^{4}$

The study of Kramer and Jacobson (1991), in particular, seems to challenge the prioritization account. Subjects were asked to report the texture of a central vertical line (i.e., whether the line was dashed or dotted) while attempting to ignore adjacent flanker lines that were themselves either dashed or dotted. The response associated with the flankers was compatible, incompatible, or neutral with respect to that required by the target. The authors varied color similarity and contour connectedness in order to manipulate whether the target and the distractors were perceived as part of the same or different objects. They found that the response compatibility effect was greater when the flankers were part of the same object as the target than when the target and the flankers were perceived as belonging to different objects.

Although this study bears a surface similarity to the present studies, it is more properly viewed as being a variant of Duncan's (1984) object-based selection paradigm. To the extent that the connecting lines caused the target and the flankers to be treated as a single object, enhancement of the flanker compatibility effect is to be expected, relative to conditions in which subjects can select the central tar- get line as a separate object, without accompanying flanker parts. Thus, like the study of Duncan, Kramer and Jacobson's (1991) object-based effects reflect attentional prioritization, and not early sensory enhancement.

In contrast, the entities that were relevant for performing the task in the present experiments were individuated letters. Despite the fact that common region can influence perceived grouping (Palmer \& Rock, 1994), the letters in the present experiments were never seen as part of a single object. Put another way, the letters were in or on the rectangles, but they were never seen as part of the rectangles. It is this fact that makes the present paradigm useful for investigating whether the "spread" of early sensory enhancement respects object boundaries. The data show that it does not.

Under what conditions, then, will object-based attentional modulation be observed? First, when different parts of a scene are selected together because they are perceived as part of a single object, those parts should be equally available for report (as in Duncan, 1984), or they should selectively interact (as in Kramer \& Jacobson, 1991). Second, when a task affords an opportunity to deploy attention to multiple locations in the scene, as in Experiment 5, locations within the same object are likely to be attended before locations that occupy different objects. In paradigms involving partially valid spatial cues (e.g., Egly et al., 1994; Moore et al., 1998), attention must be directed to both cued and uncued locations across trials, and in fact the critical evidence for object-based attentional modulation is provided by data from trials in which attention must be directed to uncued locations. In matching paradigms (e.g., Behrmann et al., 1998; Lavie \& Driver, 1996) or counting paradigms (Watson \& Kramer, 1999), attention must be directed to at least two regions in order to carry out the task. We have argued that in these paradigms, subjects may adopt a strategy that gives higher priority to locations within an already attended object over locations in other objects.

In contrast, when attention is highly focused on a single location, as in Experiments 1-4, object-based prioritization cannot exert an influence, and object-based effects are not observed. ${ }^{5}$ Similarly, when the display is brief and then masked (e.g., Moore et al., 2001), there is no time to deploy attention to more than one location, even if such a strategy might be useful. In this situation, only the effects of early sensory enhancement, which are not object based, will be observed.

\section{REFERENCES}

Avrahami, J. (1999). Objects of attention, objects of perception. Perception \& Psychophysics, 61, 1604-1612.

BAYLIS, G. C., \& Driver, J. (1992). Visual parsing and response competition: The effect of grouping factors. Perception \& Psychophysics, 51, 145-162.

Behrmann, M., Zemel, R., \& Mozer, M. (1998). Object-based attention and occlusion: Evidence from normal subjects and a computational model. Journal of Experimental Psychology: Human Perception \& Performance, 24, 1011-1036.

BerRy, G., \& KLEIN, R. (1993). Does motion-induced grouping modu- 
late the flanker compatibility effect? A failure to replicate Driver \& Baylis. Canadian Journal of Experimental Psychology, 47, 714-729. CAVE, K. R., \& Wolfe, J. M. (1990). Modeling the role of parallel processing in visual search. Cognitive Psychology, 22, 225-271.

Desimone, R, \& Duncan, J. (1995). Neural mechanisms of selective visual attention. Annual Review of Neuroscience, 18, 193-222.

Downing, C., \& Pinker, S. (1985). The spatial structure of visual attention. In M. I. Posner \& O. S. M. Marin (Eds.), Attention and performance XI (pp. 171-188). Hillsdale, NJ: Erlbaum.

Driver, J., \& BAYLIS, G. C. (1989). Movement and visual attention: The spotlight metaphor breaks down. Journal of Experimental Psychology: Human Perception \& Performance, 15, 448-456.

DunCAN, J. (1984). Selective attention and the organization of visual information. Journal of Experimental Psychology: General, 113, 501-517.

EgETH, H. E., \& YANTIS, S. (1997). Visual attention: Control, representation, and time course. Annual Review of Psychology, 48, 269-297.

Egly, R., Driver, J., \& Rafal, R. D. (1994). Shifting visual attention between objects and locations: Evidence from normal and parietal lesion subjects. Journal of Experimental Psychology: General, 123, 161-177.

Eriksen, B. A., \& Eriksen, C. W. (1974). Effects of noise letters upon the identification of a target letter in a nonsearch task. Perception \& Psychophysics, 16, 143-149.

ERIKSEN, C. W., \& Hoffman, J. E. (1972). Temporal and spatial characteristics of selective encoding from visual displays. Perception \& Psychophysics, 12, 201-204.

Harms, L., \& Bundesen, C. (1983). Color segregation and selective attention in a nonsearch task. Perception \& Psychophysics, 33, 11-19.

Hoffman, J. E., \& Nelson, B. (1981). Spatial selectivity in visual search. Perception \& Psychophysics, 30, 283-290.

Kahneman, D., \& Henik, A. (1981). Perceptual organization and attention. In M. Kubovy \& J. R. Pomerantz (Eds.), Perceptual organization (pp. 181-211). Hillsdale, NJ: Erlbaum.

KANwisher, N., \& Driver, J. (1992). Objects, attributes, and visual attention: Which what, and where. Current Directions in Psychological Science, 1, 26-31.

Kastner, S., De-Weered, P., Desimone, R., \& Ungerleider, L. G. (1998). Mechanisms of directed attention in the human extrastriate cortex as revealed by functional MRI. Science, 282, 108-111.

KrAmer, A. F., \& JACOBSON, A. (1991). Perceptual organization and focused attention: The role of objects and proximity in visual processing. Perception \& Psychophysics, 50, 267-284.

Kramer, A. F., Tham, M.-P., \& Yeh, Y.-Y. (1991). Movement and focused attention: A failure to replicate. Perception \& Psychophysics, 50, 537-546.

LABERge, D. (1983). The spatial extent of attention to letters and words. Journal of Experimental Psychology: Human Perception \& Performance, 9, 371-379.

LAMY, D. (2000). Object-based selection under focused attention: A failure to replicate. Perception \& Psychophysics, 62, 1272-1279.

Lamy, D., \& Egeth, H. (2002). Object-based selection: The role of attentional shifts. Perception \& Psychophysics, 64, 52-66.

LAVIE, N., \& Driver, J. (1996). On the spatial extent of attention in objectbased visual selection. Perception \& Psychophysics, 58, 1238-1251.

Moore, C. M., Yantis, S., \& VAughan, B. (1998). Object-based visual selection: Evidence from perceptual completion. Psychological Science, 9, 104-110.

Moore, C. M., Yantis, S., Vaughan, B., \& Handwerker, D. (2001). Functional differences between space-based and object-based visual selection. Manuscript submitted for publication.
PAlmer, S., \& Rock, I. (1994). Rethinking perceptual organization: The role of uniform connectedness. Psychonomic Bulletin \& Review, 1, 29-55.

Posner, M. I. (1980). Orienting of attention. Quarterly Journal of Experimental Psychology, 32, 3-25.

Posner, M. I., Snyder, C. R. R., \& Davidson, B. J. (1980). Attention and the detection of signals. Journal of Experimental Psychology: General, 109, 160-174.

Reynolds, J. H., Chelazzi, L., \& Desimone, R. (1999). Competitive mechanisms subserve attention in macaque areas V2 and V4. Journal of Neuroscience, 19, 1736-1753.

Rock, I., \& GutTMan, D. (1981). The effect of inattention on form perception. Journal of Experimental Psychology: Human Perception \& Performance, 7, 275-278.

Watson, S. E., \& Kramer, A. F. (1999). Object-based visual selective attention and perceptual organization. Perception \& Psychophysics, 61, 31-49.

WATT, R. J. (1984). Towards a general theory of the visual acuities for shape and spatial arrangement. Vision Research, 24, 1377-1386.

Wolfe, J. M. (1994). Guided Search 2.0: A revised model of visual search. Psychonomic Bulletin \& Review, 1, 202-238.

Yantis, S., \& Johnston, J. C. (1990). On the locus of visual selection: Evidence from focused attention tasks. Journal of Experimental Psychology: Human Perception \& Performance, 16, 135-149.

\section{NOTES}

1. Although, here and elsewhere, we discuss attentional priorization as an ordering of a strictly serial search, an equally plausible implementation would be display-wide parallel processing with different rates of information extraction according to attentional priority.

2. Moore et al. (1998) showed, however, that this effect is not merely a predisposition to follow contours, because they observed an objectspecif ic advantage even when the object in question was partly occluded by an intervening surface, thereby interrupting the contours of the attended object. This is what justifies our claim that this is an object-based scanning strategy.

3. We thank Dominique Lamy for suggesting this interpretation and experiment to us.

4. Baylis and Driver (1992; Driver \& Baylis, 1989) found that when flankers were similar in color to the target or underwent common motion with the target, they produced greater interference than otherwise, and furthermore, they found that these factors were sometimes more important than spatial proximity. The authors concluded that attention is directed not only to spatial regions, but also to perceptual groups of objects. However, the results of Baylis and Driver and of Driver and Baylis should be interpreted with caution, because they have not been consistently replicated (Berry \& Klein, 1993; Kramer, Tham, \& Yeh, 1991).

5. Lavie and Driver (1996) drew what appears to be a similar conclusion. They found that object-based effects disappeared when attention was directed to one region of the display. However, their results are difficult to reconcile with studies such as Egly et al. (1994), because their task required that attention be directed to multiple locations in the scene. Furthermore, their conclusions must be interpreted with caution, because they have not been consistently replicated (Lamy, 2000).

(Manuscript received April 3, 2000; revision accepted for publication March 26, 2001.) 